\title{
Planning Horizon for Production Inventory Models with Production Rate Dependent on Demand and Inventory Level
}

\author{
Jennifer Lin, ${ }^{1}$ Henry C. J. Chao, ${ }^{2}$ and Peterson Julian ${ }^{2}$ \\ ${ }^{1}$ Department of Transportation Logistics \& Marketing Management, Toko University, Chiayi 61363, Taiwan \\ ${ }^{2}$ Department of Traffic Science, Central Police University, Taoyuan 33334, Taiwan \\ Correspondence should be addressed to Henry C. J. Chao; una051@mail.cpu.edu.tw
}

Received 3 December 2012; Revised 1 March 2013; Accepted 2 March 2013

Academic Editor: Ching-Jong Liao

Copyright (c) 2013 Jennifer Lin et al. This is an open access article distributed under the Creative Commons Attribution License, which permits unrestricted use, distribution, and reproduction in any medium, provided the original work is properly cited.

\begin{abstract}
This paper discusses why the selection of a finite planning horizon is preferable to an infinite one for a replenishment policy of production inventory models. In a production inventory model, the production rate is dependent on both the demand rate and the inventory level. When there is an exponentially decreasing demand, the application of an infinite planning horizon model is not suitable. The emphasis of this paper is threefold. First, while pointing out questionable results from a previous study, we propose a corrected infinite planning horizon inventory model for the first replenishment cycle. Second, while investigating the optimal solution for the minimization problem, we found that the infinite planning horizon should not be applied when dealing with an exponentially decreasing demand. Third, we developed a new production inventory model under a finite planning horizon for practitioners. Numerical examples are provided to support our findings.
\end{abstract}

\section{Introduction}

Inventory models, in general, can be classified into two categories: infinite and finite planning horizon. For inventory models with the finite planning horizon, the goal is to minimize the total cost. On the other hand, without the present value, that is, not considering the time value of money, the total cost for the entire infinite planning horizon will go to infinity such that researchers are not able to compare the total cost for different inventory policies. The prevailing solution to this dilemma is to minimize the average cost of the first replenishment cycle because of a constant demand that implies an identical replenishment policy for the second replenishment cycle. As a result, the minimization of the average cost for the first replenishment cycle will lead to the optimal solution. The original paper of Wilson's EOQ model [1] is an example of an infinite planning horizon problem.

It should be noted that practitioners in previous studies seemed to randomly decide whether to use an infinite planning horizon or a finite one. That is, they make their choice either by routine experience or by referencing other studies without explaining or considering the choice that fits the characteristics of date on hand. For examples, under the assumptions of time-vary demand, production, and deterioration rate, Goyal and Giri [2] developed two models by employing different modeling approaches over an infinite planning horizon. On the other hand, Goyal's model [3] was considered as a finite planning horizon problem over time period $[0, T]$, where the replenishment cycle did not repeat itself in the same manner. It infers that each replenishment cycle within the planning horizon $[0, T]$ has different optimal solution such that the solution finding process requires the minimization of the total cost over the entire time period. For both studies, the reasoning behind the selection of either planning horizons was not explained. The purpose of this paper is to point out that in practice, some inventory models work sensibly over an infinite planning horizon. Managers under a highly competitive environment should be making correct and coherent decisions toward the development of inventory models that fit the pursuit of effective cost control. 
Many papers have also discussed production inventory models under different conditions. By viewing the production rate as a variable, Bhunia and Maiti [4] developed two inventory systems. In the first system, the production rate was dependent on the inventory level, while the production rate was dependent upon the demand in the second. Su and Lin [5] combined the two models creating a model where production rate is dependent on both inventory level and demand. Moreover, Su and Lin [5] assumed that shortages were allowed with complete backlog and an exponentially decreasing demand.

We will show that finding the minimum value of the first replenishment cycle is not reasonable with an exponentially decreasing demand since the optimal solution for the production period will go to infinity, implying that the average cost is decreasing to zero. In response, we have developed a finite planning horizon production inventory model.

There are two primary reasons that justify assuming that the demand will decrease exponentially. First, the numerous innovations in the field of technology contribute to the expedited release of new merchandises, tremendously decreasing demand for the existing products in the market. Second, rapid changes in consumer preferences also greatly impact the sales of current merchandise. For instance, less than a year after a new camera cellular phone is introduced, an even newer generation will hit the market, with higher dpi than the previous generation. As a result, the demand for the old cell phone will plunge drastically.

$\mathrm{Su}$ and Lin [5] tried to extend the findings of Bhunia and Maiti [4], but their derivation for the differential equations with boundary conditions contained questionable results. Moreover, they could not analyze how many local minimum points exist. Up to now, there have been four published papers that have referred to Su and Lin [5] in their studies, Chu and Chung [6], Alfares et al. [7], Feng and Yamashiro [8], and Kang [9]. However, none of these papers have been made aware of the fundamental flaw in Su and Lin [5].

The derivation of $\mathrm{Su}$ and $\mathrm{Lin} \mathrm{[5]} \mathrm{for} \mathrm{the} \mathrm{inventory}$ level of the third phase contained questionable results such that their findings for relations among decision variables and their objective function also had questionable results. Moreover, we showed that their model is not suitable for infinite planning horizon, and then we studied the inventory model with finite planning horizon. There are two closely related papers, Yang et al. [10] and Lin et al. [11], that are considered for the finite planning horizon. There two models are developed for the EOQ with one decision variable to show that the optimal replenishment policy is independent of the demand type. However, there are four decision variables in our EPQ inventory model. We find two relations among these decision variables of the optimal solution for the infinite planning horizon, then two independent decision variables are left. For the finite planning horizon, we proved that there is only one decision variable left. These two papers have significant contribution for the theoretical development of EOQ inventory models, but their findings cannot be applied to our EPQ inventory model.

There are four phases for an EPQ inventory model, and then we proved that there is an upper bound for the elapse time for the first phase. It is an important finding when we applied a program to locate the optimal solution. For the infinite planning horizon, we showed that four decision variables are related, so only two independent decision variables are left, and we find the relations among decision variables that reduced the tedious computation for the minimum value. For the finite planning horizon, in each replenishment cycle, we proved that there is only one independent decision variable that achieves the efficiency for computation. Our first main contribution is providing an analytical approach to solve the optimal solution such that the result from computer programs is supported by the mathematical theorem. Our second main contribution is to reduce the number of independent decision variables to its minimum such that for obtaining the optimal solution, computer programs can be executed effectively.

\section{Notation and Assumptions}

To avoid confusion, we will use the same assumptions and notation as $\mathrm{Su}$ and Lin [5]:

$\theta$ : deterioration rate,

$I_{m}$ : maximum inventory level,

$I_{b}$ : unfilled order backlog,

$C$ : setup cost for each new cycle,

$C_{d}$ : the cost of a deteriorated item,

$C_{i}$ : inventory carrying cost per unit time,

$C_{s}$ : shortage cost per unit,

$K$ : total average cost of the system.

The assumptions below are used.

(1) A single item is considered over (a) an infinite planning horizon for the first model and (b) a finite planning horizon of $T$ units of time for the second model which is subject to a constant deterioration rate.

(2) Demand rate, $D(t)$, is known and decreases exponentially so that $D(t)=A \exp (-\lambda t)$, where $A$ is the initial demand rate and $\lambda$ is the decreasing rate of demand, $0 \leq \lambda \leq 1$.

(3) $I(t)$ is the inventory level.

(4) Production rate, $P(t)$, depends on both the demand and the inventory level with $P(t)=a+b D(t)-c I(t)$, $a>0,0 \leq b<1$, and $0 \leq c<1$.

(5) Deterioration of the units is considered only after those units are received and put in inventory.

(6) There is no replacement or repair of deteriorated items.

(7) Shortages are allowed and fully backordered.

(8) Two extra conditions, $\lambda>c+\theta$ and $a \geq A$, are added (explained in Section 4). 
Remark 1. Su and Lin [5] assumed that $T$ is a prescribed period of time and denoted by $t_{4}=T$. Note that in the beginning, $\mathrm{Su}$ and $\mathrm{Lin}$ [5] tried to develop a production inventory model for a finite planning horizon, say $[0, T]$. However, during their derivation, they considered the problem of minimizing the average cost for the first replenishment cycle in the infinite planning horizon. To clearly distinguish the difference between infinite and finite planning horizon, we will separate the problem into two cases.

Case (a). We minimize the average cost of the first replenishment cycle. It is a minimization problem with an infinite planning horizon.

Case (b). We minimize the total cost over a finite planning horizon of $[0, T]$.

\section{A Review of Su and Lin [5]}

In $\mathrm{Su}$ and Lin [5], the first replenishment cycle can be divided into four phases based on the time interval:

(a) the first phase $\left[0, t_{1}\right]$ : the production dominates demand and deterioration, and the inventory level accumulates,

(b) the second phase $\left[t_{1}, t_{2}\right]$ : no production activity takes place. Demand and deterioration dominate, and so the inventory level gradually drops to zero at $t_{2}$,

(c) the third phase $\left[t_{2}, t_{3}\right]$ : no production and no deterioration take place. The shortage accumulates to $I_{b}$ at $t_{3}$,

(d) the fourth phase $\left[t_{3}, t_{4}\right]$ : the production is resumed, shortages accumulated during the third phase are fully backordered, and the inventory level returns to zero at $t_{4}$.

The differential equations developed by $\mathrm{Su}$ and Lin [5] for governing stock levels over the four different phases during the first replenishment cycle, $\left[0, t_{4}\right]$, can be expressed as follows:

$$
\begin{aligned}
\frac{d}{d t} I(t) & =P(t)-D(t)-\theta I(t) \\
& =a+(b-1) A \exp (-\lambda t)-(c+\theta) I(t), \quad 0<t<t_{1}, \\
\frac{d}{d t} I(t) & =-D(t)-\theta I(t) \\
& =-A \exp (-\lambda t)-\theta I(t), \quad t_{1}<t<t_{2}, \\
\frac{d}{d t} I(t) & =-D(t)=-A \exp (-\lambda t), \quad t_{2}<t<t_{3}, \\
\frac{d}{d t} I(t) & =P(t)-D(t) \\
& =a+(b-1) A \exp (-\lambda t)-c I(t)
\end{aligned}
$$

Under the boundary conditions,

$$
\begin{gathered}
I(0)=0, \quad I\left(t_{1}\right)=I_{m}, \quad I\left(t_{2}\right)=0, \\
I\left(t_{3}\right)=-I_{b}, \quad I\left(t_{4}\right)=0,
\end{gathered}
$$

Su and Lin [5] found that

$$
\begin{aligned}
I(t)= & \frac{a}{c+\theta}(1-\exp (-(c+\theta) t)) \\
& +\frac{A(1-b)}{\lambda-c-\theta}(\exp (-\lambda t)-\exp (-(c+\theta) t)), \quad 0 \leq t \leq t_{1}, \\
I(t)= & \frac{A \exp (-\lambda t)}{\lambda-\theta}\left(1-\exp \left(-(\lambda-\theta)\left(t_{2}-t\right)\right)\right), \\
I & t_{1} \leq t \leq t_{2}, \\
I(t)=- & \frac{a}{c}\left(\exp \left(c\left(t_{4}-t\right)\right)-1\right)(\exp (-\lambda t)-1), \quad t_{2} \leq t \leq t_{3}, \\
- & \frac{A(1-b)}{\lambda-c} \exp (-\lambda t)\left(\exp \left(-(\lambda-c)\left(t_{4}-t\right)\right)-1\right),
\end{aligned}
$$

However, the result Su and Lin [5] derived in (5) is false. The expression should be revised as

$$
I(t)=\left(\frac{A}{\lambda}\right)\left(\exp (-\lambda t)-\exp \left(-\lambda t_{2}\right)\right), \quad t_{2} \leq t \leq t_{3} .
$$

Owing to an error in (5) of their derivations for $I_{m}$ and $I_{b}$, the relation between $t_{1}$ and $t_{2}$, say $t_{2}=R\left(t_{1}\right)$, and the relation between $t_{3}$ and $t_{4}$, say $t_{3}=R\left(t_{4}\right)$, all contain questionable results. It implies that their objective function, $K\left(t_{1}, t_{2}, t_{3}, t_{4}\right)$, is also false.

$\mathrm{Su}$ and $\mathrm{Lin}$ [5] derived the expression, $K\left(t_{1}, t_{2}, t_{3}, t_{4}\right)=$ $K\left(t_{1}, t_{2}\left(t_{1}\right), t_{3}\left(t_{4}\right), t_{4}\right)$, so that the objective function has two independent variables, $t_{1}$ and $t_{4}$. They computed $\partial K / \partial t_{1}=0$ and $\partial K / \partial t_{4}=0$. However, they could not analyze whether a system that is comprised of $\partial K / \partial t_{1}=0$ and $\partial K / \partial t_{4}=0$ has solution.

\section{Our Improvement for Infinite Planning Horizon Model}

It should be pointed out that the result of (3) is based on the condition $\lambda \neq c+\theta$. On the other hand, if $\lambda=c+\theta$, (3) should 
be revised as

$$
\begin{aligned}
I(t)= & \left(\frac{a}{\lambda}\right)(1-\exp (-\lambda t)) \\
& -A t(1-b) \exp (-\lambda t), \quad 0 \leq t \leq t_{1} .
\end{aligned}
$$

Hence, if we try to provide a complete study for the production inventory model of Su and Lin [5], then our model should be divided into seven cases: case (1): $c+\theta<\lambda$, case (2): $c+\theta=\lambda$, case (3): $c<\lambda<c+\theta$, case (4): $c=\lambda$, case (5): $\theta<\lambda<c$, case (6): $\theta=\lambda$, and case (7): $\theta>\lambda$.

To focus on the investigation of a production inventory model where the production rate is dependent both on demand and inventory level, demand is exponentially decreasing, and shortages are fully backordered, we add two extra conditions: $\lambda>c+\theta$ and $a \geq A$.

The reasoning behind the addition of an extra condition, $a \geq A$, is as follows: when $t=0$, the demand rate $D(0)=A$, the inventory level $I(0)=0$, and the production rate $P(0)=$ $a+b A$. For the accumulation of inventory during the first phase $\left[0, t_{1}\right]$, it implies that $a+b A \geq A$ for $0 \leq b<1$. For the special case of $b=0$, we know that $a \geq A$ is valid. Therefore, we derive that $a \geq(1-b) A$ when $0<b<1$.

If $a<A$, then the domain of $b$ has a lower bound satisfying the expression, $b \geq 1-(a / A)$, such that the domain of $b$ should be changed from $[0,1)$ to $[1-(a / A), 1)$.

Moreover, $\mathrm{Su}$ and $\mathrm{Lin}$ [5] assumed in their numerical example that $A=200$ and $a=200$. Their assumption provides support for our extra condition of $a \geq A$. They also assumed that $\lambda=0.3, \theta=0.05$, and $c=0.2$ which provides evidence that our condition, $\lambda>c+\theta$, is reasonable. Moreover, the condition of $\lambda>c+\theta$ will focus on the development of a production inventory that is compatible with the numerical examples in Su and Lin [5] and to avoid tedious discussion for different inventory models with different relations among $\lambda, c$, and $\theta$.

Based on (3), (4), (6), and (7) and the boundary conditions of (2), we derive that

$$
\begin{aligned}
I_{m}= & \frac{a}{c+\theta}\left(1-\exp \left(-(c+\theta) t_{1}\right)\right) \\
& +\frac{A(1-b)}{\lambda-c-\theta}\left(\exp \left(-\lambda t_{1}\right)-\exp \left(-(c+\theta) t_{1}\right)\right) \\
= & \frac{A \exp \left(-\lambda t_{1}\right)}{\lambda-\theta}\left(1-\exp \left(-(\lambda-\theta)\left(t_{2}-t_{1}\right)\right)\right), \\
I_{b}= & \left(\frac{A}{\lambda}\right)\left(\exp \left(-\lambda t_{2}\right)-\exp \left(-\lambda t_{3}\right)\right) \\
= & \left(\frac{a}{c}\right)\left(\exp \left(c\left(t_{4}-t_{3}\right)\right)-1\right) \\
& +\frac{A(1-b)}{\lambda-c}\left(\exp \left((c-\lambda)\left(t_{4}-t_{3}\right)\right)-1\right) \exp \left(-\lambda t_{3}\right) .
\end{aligned}
$$

From (9), we find the relation between $t_{1}$ and $t_{2}$ and then the relation among $t_{2}, t_{3}$, and $t_{4}$ :

$$
\begin{aligned}
t_{2}=\frac{1}{\theta-\lambda} \ln [ & \exp \left((\theta-\lambda) t_{1}\right) \\
& -\frac{a(\lambda-\theta)}{A(c+\theta)}\left(\exp \left(\theta t_{1}\right)-\exp \left(-c t_{1}\right)\right) \\
& -\frac{(\lambda-\theta)(1-b)}{\lambda-c-\theta} \\
& \left.\times\left(\exp \left((\theta-\lambda) t_{1}\right)-\exp \left(-c t_{1}\right)\right)\right] \\
\frac{A}{\lambda}(\exp ( & \left.\left.-\lambda t_{2}\right)-\exp \left(-\lambda t_{3}\right)\right)+\frac{a}{c}+\frac{A(1-b)}{\lambda-c} \exp \left(-\lambda t_{3}\right) \\
=( & \left.\frac{a}{c}+\frac{A(1-b)}{\lambda-c} \exp \left(-\lambda t_{4}\right)\right) \exp \left(c\left(t_{4}-t_{3}\right)\right) .
\end{aligned}
$$

We will simplify a four-variable problem, $t_{1}, t_{2}, t_{3}$, and $t_{4}$, to a two-variable problem of $t_{1}$ and $t_{3}$. During $\left[0, t_{1}\right]$, production, demand, and deterioration interact with each other to accumulate items that will be consumed and thus deteriorate during $\left[t_{1}, t_{2}\right]$ such that, trivially, $t_{2}-t_{1}$ is dependent on $t_{1}$. We will derive the detailed relation between $t_{2}-t_{1}$ and $t_{1}$, that is, $t_{2}$ and $t_{1}$. During $\left[t_{2}, t_{3}\right]$, the shortages will accumulate to be backlogged during $\left[t_{3}, t_{4}\right]$ so that naturally $t_{4}-t_{3}$ is dependent on $t_{3}-t_{2}$. We will derive the detailed relation between $t_{4}-t_{3}$ and $t_{3}-t_{2}$, which is the relation of (i) $t_{4}$ and (ii) $t_{3}$ with $t_{2}$. Due to the fact that demand is varied, $t_{2}$ will influence the shortage during $\left[t_{2}, t_{3}\right]$.

In the following, we will prove that $t_{2}$ can be uniquely decided if $t_{1}$ is given. When $t_{1}$ and $t_{2}$ are given, by using the relation in (11), the unique value of $t_{4}$ can be derived if $t_{3}$ is also given. Hence, we will simplify a four-variable problem to a two-variable problem. Let us rewrite (10) as

$$
\begin{aligned}
1-\exp \left(-(\lambda-\theta)\left(t_{2}-t_{1}\right)\right) \\
=\frac{a(\lambda-\theta)}{A(c+\theta)}\left[\exp \left(\lambda t_{1}\right)-\exp \left((\lambda-c-\theta) t_{1}\right)\right] \\
\quad+\frac{(\lambda-\theta)(1-b)}{\lambda-c-\theta}\left[1-\exp \left((\lambda-c-\theta) t_{1}\right)\right] .
\end{aligned}
$$

We tried to find the condition of $t_{1}$ under which there is a solution to $t_{2}$ with $t_{2} \geq t_{1}$, satisfying (12). For the later discussion, given that $t_{1}$, we denote the unique solution of $t_{2}$ that satisfies (12) as $t_{2}\left(t_{1}\right)$. We will prove that the feasible domain of $t_{1}$ is bounded, guaranteeing the existence of $t_{2}$.

Motivated by (12), we assume the following auxiliary function, $g\left(t_{1}\right)$, to be

$$
\begin{aligned}
g\left(t_{1}\right)= & \frac{a(\lambda-\theta)}{A(c+\theta)}\left[\exp \left(\lambda t_{1}\right)-\exp \left((\lambda-c-\theta) t_{1}\right)\right] \\
& +\frac{(\lambda-\theta)(1-b)}{\lambda-c-\theta}\left[1-\exp \left((\lambda-c-\theta) t_{1}\right)\right] .
\end{aligned}
$$


Taking the derivative of $g\left(t_{1}\right)$ with respect to $t_{1}$ yields

$$
\begin{aligned}
& \frac{d}{d t_{1}} g\left(t_{1}\right) \\
& =\frac{a(\lambda-\theta)}{A(c+\theta)}\left[\lambda \exp \left(\lambda t_{1}\right)-(\lambda-c-\theta) \exp \left((\lambda-c-\theta) t_{1}\right)\right] \\
& \quad-(\lambda-\theta)(1-b) \exp \left((\lambda-c-\theta) t_{1}\right)
\end{aligned}
$$

Under the conditions $a \geq A$ and $\lambda>c+\theta$, it follows that

$$
\begin{aligned}
& \frac{d}{d t_{1}} g\left(t_{1}\right) \\
& \geq \frac{(\lambda-\theta)}{(c+\theta)}\left[\lambda \exp \left(\lambda t_{1}\right)-(\lambda-c-\theta) \exp \left((\lambda-c-\theta) t_{1}\right)\right] \\
& \quad-(\lambda-\theta) \exp \left((\lambda-c-\theta) t_{1}\right) \\
& =\frac{\lambda(\lambda-\theta)}{c+\theta}\left[\exp \left(\lambda t_{1}\right)-\exp \left((\lambda-c-\theta) t_{1}\right)\right]>0
\end{aligned}
$$

showing that $g\left(t_{1}\right)$ is an increasing function from $g(0)=0$ to $\lim _{t_{1} \rightarrow \infty} g\left(t_{1}\right)=\infty$, since

$$
\begin{aligned}
\lim _{t_{1} \rightarrow \infty} \frac{g\left(t_{1}\right)}{\exp \left((\lambda-c-\theta) t_{1}\right)} \\
=\lim _{t_{1} \rightarrow \infty}\left[\frac{a(\lambda-\theta)}{A(c+\theta)}\left(\exp \left((c+\theta) t_{1}\right)-1\right)\right. \\
\left.\quad+\frac{(\lambda-\theta)(1-b)}{\lambda-c-\theta}\left(\exp \left(-(\lambda-c-\theta) t_{1}\right)-1\right)\right]
\end{aligned}
$$$$
=\infty \text {. }
$$

There is a unique point, say $t_{1}^{\#}$, that satisfies $g\left(t_{1}^{\#}\right)=1$.

From (3) and (13), we have

$$
\frac{\lambda-\theta}{A} \exp \left(\lambda t_{1}^{\#}\right) I\left(t_{1}^{\#}\right)=g\left(t_{1}^{\#}\right)=1 .
$$

From $\lambda>c+\theta$, the inequality, $\lambda>\theta$, is held. It follows that

$$
I\left(t_{1}^{\#}\right)=\frac{A}{\lambda-\theta} \exp \left(-\lambda t_{1}^{\#}\right) .
$$

By referring to (12), we obtain

$$
g\left(t_{1}^{\#}\right)=1=1-\exp \left(-(\lambda-\theta)\left(t_{2}\left(t_{1}^{\#}\right)-t_{1}^{\#}\right)\right) .
$$

According to (19), we showed that $t_{2}\left(t_{1}^{\#}\right)-t_{1}^{\#}$ must go to $\infty$ so that $t_{2}\left(t_{1}^{\#}\right)$ will go to $\infty$ as well. We will express the result as $\lim _{t_{1} \rightarrow t_{1}^{\sharp}} t_{2}\left(t_{1}\right)=\infty$ and summarize our findings in the next lemma.

Lemma 2. $I\left(t_{1}^{\#}\right)=(A /(\lambda-\theta)) e^{-\lambda t_{1}^{\#}}$ and $\lim _{t_{1} \rightarrow t_{1}^{*}} t_{2}\left(t_{1}\right)=\infty$.
From Lemma 2, we know that the feasible domain of $t_{1}$ should be set as

$$
0 \leq t_{1}<t_{1}^{\#} \text {. }
$$

Given $t_{1}$, with $t_{1}<t_{1}^{\#}$, then $g\left(t_{1}\right)<1$ so that there is a unique $t_{2}$, say $t_{2}\left(t_{1}\right)$, that satisfies

$$
1-\exp \left(-(\lambda-\theta)\left(t_{2}\left(t_{1}\right)-t_{1}\right)\right)=g\left(t_{1}\right) .
$$

We may explicitly express $t_{2}\left(t_{1}\right)$ as

$$
\begin{aligned}
& t_{2}\left(t_{1}\right) \\
& \begin{aligned}
=t_{1}-\frac{1}{\lambda-\theta} \ln [ & 1-\frac{a(\lambda-\theta)}{A(c+\theta)} \\
& \times\left[\exp \left(\lambda t_{1}\right)-\exp \left((\lambda-c-\theta) t_{1}\right)\right] \\
& \left.-\frac{(\lambda-\theta)(1-b)}{\lambda-c-\theta}\left[1-\exp \left((\lambda-c-\theta) t_{1}\right)\right]\right] .
\end{aligned}
\end{aligned}
$$

We will summarize our findings in the following lemma.

Lemma 3. If $t_{1}<t_{1}^{\#}$, then there is a unique $t_{2}$, say $t_{2}\left(t_{1}\right)$, as in (22) so that (10) is satisfied.

Next, we consider the relation among $t_{2}, t_{3}$, and $t_{4}$ by rewriting (11) as

$$
\begin{gathered}
{\left[\frac{A}{\lambda}\left(\exp \left(-\lambda t_{2}\right)-\exp \left(-\lambda t_{3}\right)\right)+\frac{a}{c}+\frac{A(1-b)}{\lambda-c} \exp \left(-\lambda t_{3}\right)\right]} \\
\quad \times \exp \left(c t_{3}\right) \\
=\left(\frac{a}{c}+\frac{A(1-b)}{\lambda-c} \exp \left(-\lambda t_{4}\right)\right) \exp \left(c t_{4}\right) .
\end{gathered}
$$

Motivated by (23), we assume the following auxiliary function:

$$
f\left(t_{4}\right)=\left(\frac{a}{c}+\frac{A(1-b)}{\lambda-c} \exp \left(-\lambda t_{4}\right)\right) \exp \left(c t_{4}\right) .
$$

We find that

$$
\begin{aligned}
f^{\prime}\left(t_{4}\right) & =\exp \left(c t_{4}\right)\left[a-A(1-b) \exp \left(-\lambda t_{4}\right)\right] \\
& >a-A(1-b) \geq 0 .
\end{aligned}
$$

Under our assumptions of $a \geq A$ and $0 \leq b<1$, it can be inferred that $f\left(t_{4}\right)$ increases from $f\left(t_{3}\right)=(a / c) \exp \left(c t_{3}\right)+$ $(A(1-b) /(\lambda-c)) \exp \left((c-\lambda) t_{3}\right)$ to $\lim _{t_{4} \rightarrow \infty} f\left(t_{4}\right)=\infty$.

The relation below,

$$
\begin{gathered}
{\left[\frac{A}{\lambda}\left(\exp \left(-\lambda t_{2}\right)-\exp \left(-\lambda t_{3}\right)\right)+\frac{a}{c}+\frac{A(1-b)}{\lambda-c} \exp \left(-\lambda t_{3}\right)\right]} \\
\quad \times \exp \left(c t_{3}\right) \\
\geq \frac{a}{c} \exp \left(c t_{3}\right)+\frac{A(1-b)}{\lambda-c} \exp \left((c-\lambda) t_{3}\right)=f\left(t_{3}\right),
\end{gathered}
$$


holds since $t_{2} \leq t_{3}$. Therefore, if $t_{1}$ and $t_{3}$ are decided, with the restriction $t_{1}<t_{1}^{\#}$, then there is a unique $t_{2}\left(t_{1}\right)$ that satisfies (10). Also, from (26) and the increasing function $f\left(t_{4}\right)$, we know that for a given $t_{3}$ under the condition, $t_{2}\left(t_{1}\right) \leq t_{3}$, there is a unique point explicitly denoted as $t_{4}\left(t_{1}, t_{2}\left(t_{1}\right), t_{3}\right)$, simply say $t_{4}$, that satisfies the condition, $t_{4} \geq t_{3}$, such that the following expression,

$$
\begin{aligned}
& {\left[\frac{A}{\lambda}\left(\exp \left(-\lambda t_{2}\right)-\exp \left(-\lambda t_{3}\right)\right)+\frac{a}{c}+\frac{A(1-b)}{\lambda-c} \exp \left(-\lambda t_{3}\right)\right]} \\
& \quad \times \exp \left(c t_{3}\right) \\
& =f\left(t_{4}\right),
\end{aligned}
$$

satisfies (23). We will summarize our results in the next lemma.

Lemma 4. If $t_{1}$ and $t_{3}$ are given with $t_{1}<t_{1}^{\#}$ and $t_{2}\left(t_{1}\right) \leq$ $t_{3}$, then there is a unique $t_{4}$, denoted as $t_{4}\left(t_{1}, t_{2}\left(t_{1}\right), t_{3}\right)$ that satisfies (11).

Up to this point, the corrected objective function below can be provided as

$$
\begin{aligned}
& K\left(t_{1}, t_{2}, t_{3}, t_{4}\right) \\
& =\left\{\frac{a}{c+\theta}\left(t_{1}+\frac{\exp \left(-(c+\theta) t_{1}\right)-1}{c+\theta}\right)+\frac{A(1-b)}{\lambda-c-\theta}\right. \\
& \times\left(\frac{\exp \left(-(c+\theta) t_{1}\right)-1}{c+\theta}-\frac{\exp \left(-\lambda t_{1}\right)-1}{\lambda}\right) \\
& +\frac{A}{\lambda-\theta}\left(\frac{\exp \left(-\lambda t_{1}\right)-\exp \left(-\lambda t_{2}\right)}{\lambda}+\exp \left(-(\lambda-\theta) t_{2}\right)\right. \\
& \left.\left.\times \frac{\exp \left(-\theta t_{2}\right)-\exp \left(-\theta t_{1}\right)}{\theta}\right)\right\} \frac{\theta C_{d}+C_{i}}{t_{4}} \\
& +\frac{C_{s}}{t_{4}}\left\{\frac{A}{\lambda^{2}}\left(\exp \left(-\lambda t_{3}\right)-\exp \left(-\lambda t_{2}\right)\right)\right. \\
& +\frac{A}{\lambda} \exp \left(-\lambda t_{2}\right)\left(t_{3}-t_{2}\right)-\frac{a}{c^{2}}\left(1-\exp \left(c\left(t_{4}-t_{3}\right)\right)\right) \\
& -\frac{a}{c}\left(t_{4}-t_{3}\right)+\frac{A(1-b)}{\lambda-c} \\
& \times\left(\exp \left(-(\lambda-c) t_{4}\right)\left(\frac{\exp \left(-c t_{3}\right)-\exp \left(-c t_{4}\right)}{c}\right)\right. \\
& \left.\left.+\frac{\exp \left(-\lambda t_{4}\right)-\exp \left(-\lambda t_{3}\right)}{\lambda}\right)\right\}+\frac{C}{t_{4}},
\end{aligned}
$$

with the conditions $0<t_{1} \leq t_{2} \leq t_{3} \leq t_{4}$.

Given $t_{1}$, with $t_{1}<t_{1}^{\#}$ and $(22), t_{2}\left(t_{1}\right)$ can be derived. Given a $t_{3}$ that satisfies $t_{2}\left(t_{1}\right) \leq t_{3}$, then, by (23), $t_{4}$ can also be obtained. We have learned from above discussion that only $t_{1}$ and $t_{3}$ are independent variables.
TABLE 1: The results for average cost of the first cycle for infinite planning horizon.

\begin{tabular}{lcccc}
\hline$t_{1}$ & $t_{2}$ & $t_{3}$ & $t_{4}$ & $K\left(t_{1}, t_{2}, t_{3}, t_{4}\right)$ \\
\hline 1.4683 & 2.2261 & 2.3148 & 2.3885 & 88.8785 \\
2.4280 & 4.6512 & 4.9220 & 4.9876 & 102.8907 \\
3.2554 & 9.1627 & 9.9433 & 9.9794 & 108.9192 \\
3.6192 & 17.6260 & 19.9899 & 19.9985 & 77.4016 \\
3.6626 & 25.4135 & 29.9900 & 29.9912 & 54.0973 \\
3.6695 & 37.6621 & 477.5669 & 477.5670 & 3.4965 \\
3.6696 & 39.0597 & 1745.8406 & 1745.8407 & 0.9889 \\
3.6698 & 46.3253 & 5929.4429 & 5929.4434 & 0.2818 \\
3.6698 & 46.3253 & 14903.3271 & 14903.3281 & 0.1158 \\
3.6698 & 46.3253 & 19998.2832 & 19998.2852 & 0.0879 \\
\hline
\end{tabular}

Hence, the problem becomes the minimization of $K\left(t_{1}, t_{2}\left(t_{1}\right), t_{3}, t_{4}\left(t_{1}, t_{2}\left(t_{1}\right), t_{3}\right)\right)$ under two restrictions:

$$
\begin{gathered}
t_{1}<t_{1}^{\#}, \\
t_{2}\left(t_{1}\right) \leq t_{3} .
\end{gathered}
$$

We have derived a two-variable minimum problem of $t_{1}$ and $t_{3}$ under the conditions of (29), for the infinite horizon minimum cost inventory model. The findings are concluded in the next theorem.

Theorem 5. For the production inventory model with infinite planning horizon, if one minimizes the average cost for the first replenishment cycle, then there are two necessary conditions, $t_{1}<t_{1}^{\#}$ and $t_{2}\left(t_{1}\right) \leq t_{3}$, for the production inventory model of Su and Lin [5].

\section{Numerical Examples for Infinite Planning Horizon Inventory Model}

We will employ the same numerical examples as $\mathrm{Su}$ and Lin [5] for comparison purposes where $A=200, \lambda=0.3, \theta=$ 0.05, $C=100, C_{d}=3, C_{s}=10, C_{i}=1, a=200, b=0.2$, and $c=0.2$. Some computation results are showed in Table 1 with $t_{1}^{\#}=3.6699$ arranged according to a sequence of different values of $t_{3}$.

From the numerical examples in Table 1, it reveals that if we prolong the replenishment cycle, then the average cost will eventually decrease. The rationale is that with a negatively exponential demand function, the market demand will dramatically decrease, especially in a longer inventory horizon, which will in turn significantly bring down the corresponding average holding and shortage costs. On the other hand, when we prolong the shortage phase with $t_{3}=$ 19998.2832 (the personal computer's computational limit) in order to reduce the average cost, the ordinary customers may lose patience when waiting for the backorder. Hence, a full backorder cannot be performed. In other words, it is impossible to simultaneously achieve full backorder and minimize average inventory cost. We may conclude that for the negatively exponential demand, $D(t)=A \exp (-\lambda t)$, the infinite planning horizon production inventory model is not 
adequate. Therefore, we stop the discussion of case (a) in the infinite planning horizon.

\section{Our Proposed Production Inventory Model with Finite Planning Horizon}

Next, we consider case (b) with a finite planning horizon, denoted by $[0, T]$. To simplify the discussion, we assume that there is one replenishment cycle during the finite planning horizon. Our results can be easily extended to several replenishment cycles. In this setting, (28) should be revised as follows:

$$
\begin{aligned}
& K\left(t_{1}, t_{2}, t_{3}, t_{4}=T\right) \\
& =\left\{\frac{a}{c+\theta}\left(t_{1}+\frac{\exp \left(-(c+\theta) t_{1}\right)-1}{c+\theta}\right)+\frac{A(1-b)}{\lambda-c-\theta}\right. \\
& \quad \times\left(\frac{\exp \left(-(c+\theta) t_{1}\right)-1}{c+\theta}-\frac{\exp \left(-\lambda t_{1}\right)-1}{\lambda}\right) \\
& +\frac{A}{\lambda-\theta}\left(\frac{\exp \left(-\lambda t_{1}\right)-\exp \left(-\lambda t_{2}\right)}{\lambda}+\exp \left(-(\lambda-\theta) t_{2}\right)\right. \\
& +\frac{C_{s}}{T}\left\{\frac{A}{\lambda^{2}}\left(\exp \left(-\lambda t_{3}\right)-\exp \left(-\lambda t_{2}\right)\right)\right. \\
& \left.+\frac{A}{\lambda} \exp \left(-\lambda t_{2}\right)\left(t_{3}-t_{2}\right)-\frac{a}{c^{2}}\left(1-\exp \left(-\theta t_{2}\right)-\exp \left(-\theta t_{1}\right)\right)\right\} \frac{\theta C_{d}+C_{i}}{T} \\
& \quad+\frac{a}{c}\left(T-t_{3}\right)+\frac{A(1-b)}{\lambda-c} \\
& \times(\exp (-(\lambda-c) T)) \\
& \left.\left.+\frac{\exp (-\lambda T)-\exp \left(-\lambda t_{3}\right)}{\lambda}\right)\right\}+\frac{C}{T} .
\end{aligned}
$$

Here, we will derive a stronger condition than $t_{1}<t_{1}^{\#}$ for the feasible domain of $t_{1}$. For a given $t_{4}$, from (10), since $t_{2}\left(t_{1}\right)$ is an increasing function of $t_{1}$, there is a unique point, say $t_{1}^{\wedge}\left(t_{4}\right)$ with $t_{2}\left(t_{1}^{\wedge}\left(t_{4}\right)\right)=t_{4}$. Under the condition

$$
t_{1}<t_{1}^{\wedge}\left(t_{4}\right)
$$

the desired result $t_{2}\left(t_{1}\right)<t_{4}$ is achieved, since $t_{2}\left(t_{1}\right)$ is an increasing function of $t_{1}$.

Lemma 6. For a given $t_{4}$, the feasible domain of $t_{1}$ is $\left[0, t_{1}^{\wedge}\left(t_{4}\right)\right)$, implying that $t_{2}\left(t_{1}\right)<t_{4}$ with $t_{2}\left(t_{1}^{\wedge}\left(t_{4}\right)\right)=t_{4}$.

In the following, when $t_{4}$ is given, if we take a $t_{1}$ that satisfies (31), then we will prove that there is a unique $t_{3}$ that satisfies (23).
Based on (23), let us assume other two auxiliary functions, $k(t)$ and $h\left(t_{3}\right)$, where

$$
\begin{aligned}
k(t)= & \frac{a}{c} \exp (c t) \\
& +\frac{A(1-b)}{\lambda-c} \exp (-(\lambda-c) t), \quad \text { for } t_{2} \leq t \leq T .
\end{aligned}
$$

With a restricted domain, $k(t)$ is related to our previous auxiliary function $f\left(t_{4}\right)$ of $(24)$ and

$$
\begin{aligned}
& h\left(t_{3}\right) \\
& \qquad=\left[\frac{A}{\lambda}\left(\exp \left(-\lambda t_{2}\right)-\exp \left(-\lambda t_{3}\right)\right)+\frac{a}{c}\right. \\
& \left.\quad+\frac{A(1-b)}{\lambda-c} \exp \left(-\lambda t_{3}\right)\right] \exp \left(c t_{3}\right), \quad \text { for } t_{2} \leq t_{3} \leq T .
\end{aligned}
$$

From $(d / d t) k(t)=a \exp (c t)-A(1-b) \exp (-(\lambda-c) t)>0$, under the conditions $a \geq A$ and $0 \leq b<1, k(t)$ is an increasing function which implies that

$$
\begin{aligned}
k\left(t_{2}\right) & =\frac{a}{c} \exp \left(c t_{2}\right)+\frac{A(1-b)}{\lambda-c} \exp \left(-(\lambda-c) t_{2}\right)<k(T) \\
& =\frac{a}{c} \exp (c T)+\frac{A(1-b)}{\lambda-c} \exp (-(\lambda-c) T)
\end{aligned}
$$

On the other hand, the expression

$$
\begin{aligned}
& \frac{d}{d t_{3}} h\left(t_{3}\right) \\
& \quad \begin{array}{l}
\frac{A}{\lambda}\left[\lambda \exp \left(-(\lambda-c) t_{3}\right)\right. \\
\left.\quad+c\left(\exp \left(-c t_{2}\right)-\exp \left(-\lambda t_{3}\right)\right) \exp \left(c t_{3}\right)\right] \\
+a \exp \left(c t_{3}\right)-A(1-b) \exp \left((c-\lambda) t_{3}\right)>0
\end{array}
\end{aligned}
$$

shows that $h\left(t_{3}\right)$ is an increasing function. If we apply (33) and (34), then $h\left(t_{3}\right)$ increases from

$$
h\left(t_{2}\right)=k\left(t_{2}\right)<k(T),
$$

to

$$
h(T)=\frac{A}{\lambda}\left(\exp \left(-\lambda t_{2}\right)-\exp (-\lambda T)\right)+k(T)>k(T) .
$$

Therefore, there is a unique point, say $t_{3}\left(t_{2}\right)$, that satisfies

$$
h\left(t_{3}\left(t_{2}\right)\right)=k(T) \text {. }
$$

When $t_{4}=T$ is given, based on the previous discussion, if $t_{1}$ is given with $t_{1}<t_{1}^{\wedge}\left(t_{4}\right)$, then we have $t_{2}\left(t_{1}\right)<t_{4}$. From (38), there exists a unique point, $t_{3}\left(t_{2}\right)$, with $h\left(t_{3}\left(t_{2}\left(t_{1}\right)\right)\right)=$ $K(T)$ such that $t_{2}\left(t_{1}\right), t_{3}\left(t_{2}\left(t_{1}\right)\right)$, and $t_{4}$ satisfy (23). Hence, for a finite-horizon minimum cost inventory model, we have simplified a four-variable problem to a one-variable problem. 
Hence, in the following, if we only consider those $t_{1} s$ that satisfy the condition of (31), then

$$
t_{2}\left(t_{1}\right)<t_{4}
$$

By (23) and (38), the relation, $t_{3}=t_{3}\left(t_{2}\right)$, implies that

$$
t_{4}\left(t_{1}, t_{2}\left(t_{1}\right), t_{3}\left(t_{2}\left(t_{1}\right)\right)\right)=t_{4},
$$

where $t_{4}\left(t_{1}, t_{2}\left(t_{1}\right), t_{3}\right)$, defined in Lemma 4 , satisfies (11).

The objective function becomes a one-variable problem

$$
\left.\begin{array}{l}
K\left(t_{1}\right) \\
=K\left(t_{1}, t_{2}\left(t_{1}\right), t_{3}\left(t_{2}\left(t_{1}\right)\right), t_{4}=T\right) \\
=\frac{C}{T}+\frac{\theta C_{d}+C_{i}}{T} \\
\times\left\{\frac{a}{c+\theta}\left(t_{1}+\frac{\exp \left(-(c+\theta) t_{1}\right)-1}{c+\theta}\right)\right. \\
+\frac{A(1-b)}{\lambda-c-\theta}\left(\frac{\exp \left(-(c+\theta) t_{1}\right)-1}{c+\theta}\right. \\
+\frac{A}{\lambda-\theta}\left(\frac{\exp \left(-\lambda t_{1}\right)-\exp \left(-\lambda t_{2}\right)}{\lambda}\right. \\
+\frac{C_{3}}{T}\left\{\frac{A}{\lambda^{2}}\left(\exp \left(-\lambda t_{3}\right)-\exp \left(-\lambda t_{2}\right)\right)\right. \\
\left.+\frac{\exp (-\lambda T)-\exp \left(-\lambda t_{3}\right)}{\lambda}\right) \\
+\exp \left(-(\lambda-\theta) t_{2}\right) \\
\left.\left.\times \frac{\exp \left(-\theta t_{2}\right)-\exp \left(-\theta t_{1}\right)}{\theta}\right)\right\}
\end{array}\right\}
$$

We will summarize our findings in the next theorem.

Theorem 7. For the production inventory model with the finite planning horizon, $\left[0, t_{4}\right]$, if one only considers one replenishment cycle, there is a natural restriction $t_{1}<t_{1}^{\wedge}\left(t_{4}\right)$, creating a one-variable minimum problem.
From Theorem 7, computer program as MathCAD can be adopted to locate the optimal solution. We may point out that the benefits to the simplified production inventory model that we have proposed include (a) easy to use for decision makers, (b) reduction of the solution space (computation time) in determining the parameter setting, and (c) reduction of the model complexity.

\section{Numerical Example for the Finite Planning Horizon}

For the finite planning horizon production inventory model with the same data, $A=200, \lambda=0.3, \theta=0.05, C=100$, $C_{d}=3, C_{s}=10, C_{i}=1, a=200, b=0.2, c=0.2$, and $T=2$, we find the optimal solution, $t_{1}^{*}=1.2742$. With (12), it shows that $t_{2}^{*}=1.8620$. With (38), it shows that $t_{3}^{*}=1.9306$. Finally, with (41), we find that the minimum cost is $K\left(t_{1}^{*}, t_{2}^{*}, t_{3}^{*}, t_{4}^{*}\right)=89.7151$. The above discussion is based on the preset condition that there is one replenishment cycle. However, under the multiple replenishment cycles, the total setup cost will be at least 200, and then the average cost during $[0,2]$ is more than one hundred that is larger than the result of one replenishment cycle. It specifies that the average cost for multiple replenishment cycles is much larger than that of one replenishment cycle. Hence, for this numerical example, we only consider one replenishment cycle.

Particle swarm optimization is applied to check our findings. Both approaches have the same optimal solution.

\section{Conclusion and Further Direction}

We have shown that with an exponentially decreasing demand, the goals of simultaneously minimizing the average cost for the first replenishment cycle and fully backordering the shortage items cannot be applied for an infinitehorizon minimum cost inventory model. The result of our investigation explicitly reveals that using the infinite planning horizon model is inappropriate in practice. For the finite planning horizon, we have shown that the four-phase production inventory model can be converted to a single variable problem in order to find the minimum solution. Our study not only provides a sound operational formulation but also offers a practical and efficient approach in the location of the optimal solution.

The study we have carried out can probably be viewed as the first attempt to solve a finite planning horizon production inventory model. In the future, it would be interesting to show that our objective function is convex to ensure the existence of a local minimum. Moreover, the issues of how to decide the optimal solution under several replenishment cycles and how to verify the convexity of the minimum value under multiple replenishment cycles deserve further study.

\section{Acknowledgments}

This paper is partially supported by the National Science Council of Taiwan, Taiwan, with Grant NSC 101-2410-H-01502. The authors want to express their gratitude to Sophia Liu 
(sophia0liu@hotmail.com) for her English revisions of their paper and Professor Shih-Wei Lin for his help with particle swarm optimization.

\section{References}

[1] R. H. Wilson, "A scientific routine for stock control," Harvard Business Review, vol. 13, no. 1, pp. 116-128, 1934.

[2] S. K. Goyal and B. C. Giri, "The production-inventory problem of a product with time varying demand, production and deterioration rates," European Journal of Operational Research, vol. 147, no. 3, pp. 549-557, 2003.

[3] S. K. Goyal, "Economic packaging frequency for finite horizon inventory problems," International Journal of Production Research, vol. 13, no. 4, pp. 383-389, 1975.

[4] A. K. Bhunia and M. Maiti, "Deterministic inventory models for variable production," Journal of the Operational Research Society, vol. 48, no. 2, pp. 221-224, 1997.

[5] C. T. Su and C. W. Lin, "A production inventory model which considers the dependence of production rate on demand and inventory level," Production Planning and Control, vol. 12, no. 1, pp. 69-75, 2001.

[6] P. Chu and K.-J. Chung, "The sensitivity of the inventory model with partial backorders," European Journal of Operational Research, vol. 152, no. 1, pp. 289-295, 2004.

[7] H. K. Alfares, S. N. Khursheed, and S. M. Noman, "Integrating quality and maintenance decisions in a production-inventory model for deteriorating items," International Journal of Production Research, vol. 43, no. 5, pp. 899-911, 2005.

[8] D. Z. Feng and M. Yamashiro, "Optimal production policy for a two-stage production system under lumpy demand," in Proceedings of the 18th Australian Joint conference on Advances in Artificial Intelligence (AI '05), vol. 3809 of Lecture Notes in Computer Science, pp. 1173-1179, 2005.

[9] H. Y. Kang, "Optimal replenishment policies for deteriorating control wafers inventory," International Journal of Advanced Manufacturing Technology, vol. 35, no. 7-8, pp. 736-744, 2008.

[10] G. K. Yang, R. Lin, J. Lin, K. C. Hung, P. Chu, and W. Chouhuang, "Note on inventory models with Weibull distribution deterioration," Production Planning and Control, vol. 22, no. 4, pp. 437-444, 2011.

[11] J. Lin, H. Chao, and P. Julian, "A demand independent inventory model," Yugoslav Journal of Operations Research, vol. 22, no. 2, p. 21, 2013. 


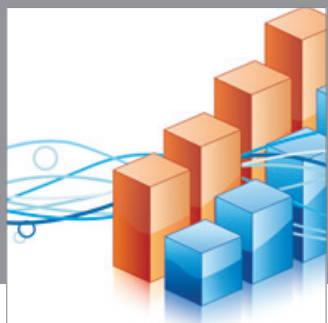

Advances in

Operations Research

mansans

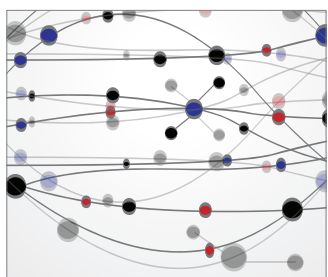

The Scientific World Journal
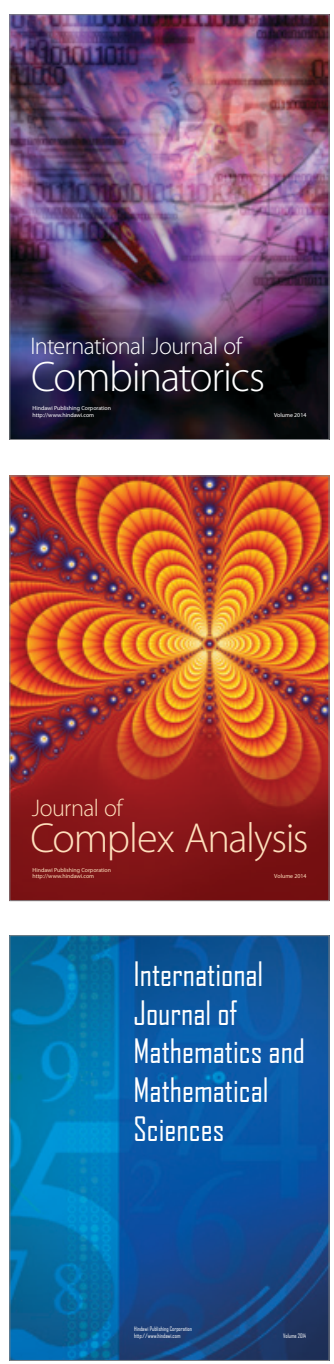
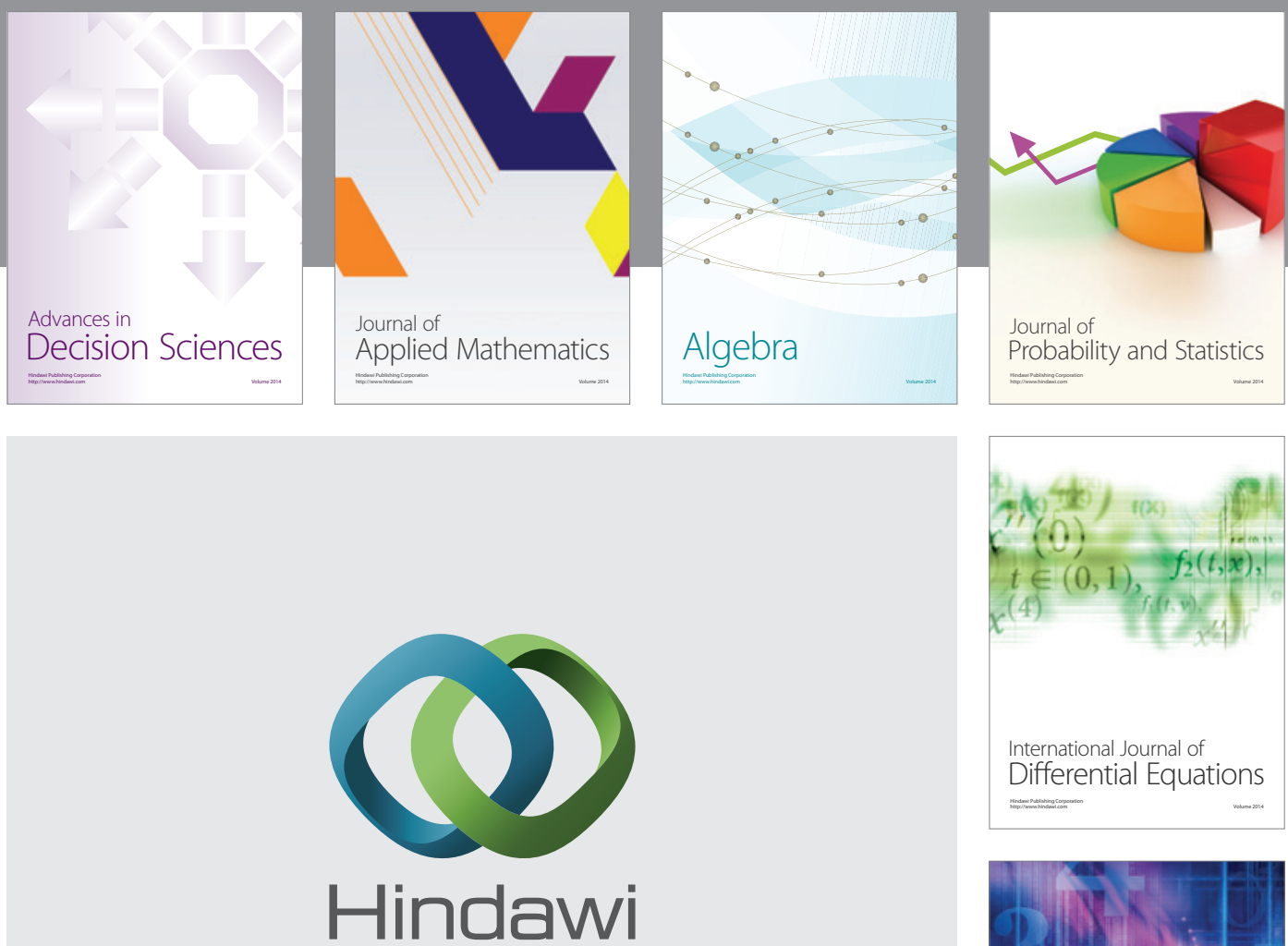

Submit your manuscripts at http://www.hindawi.com
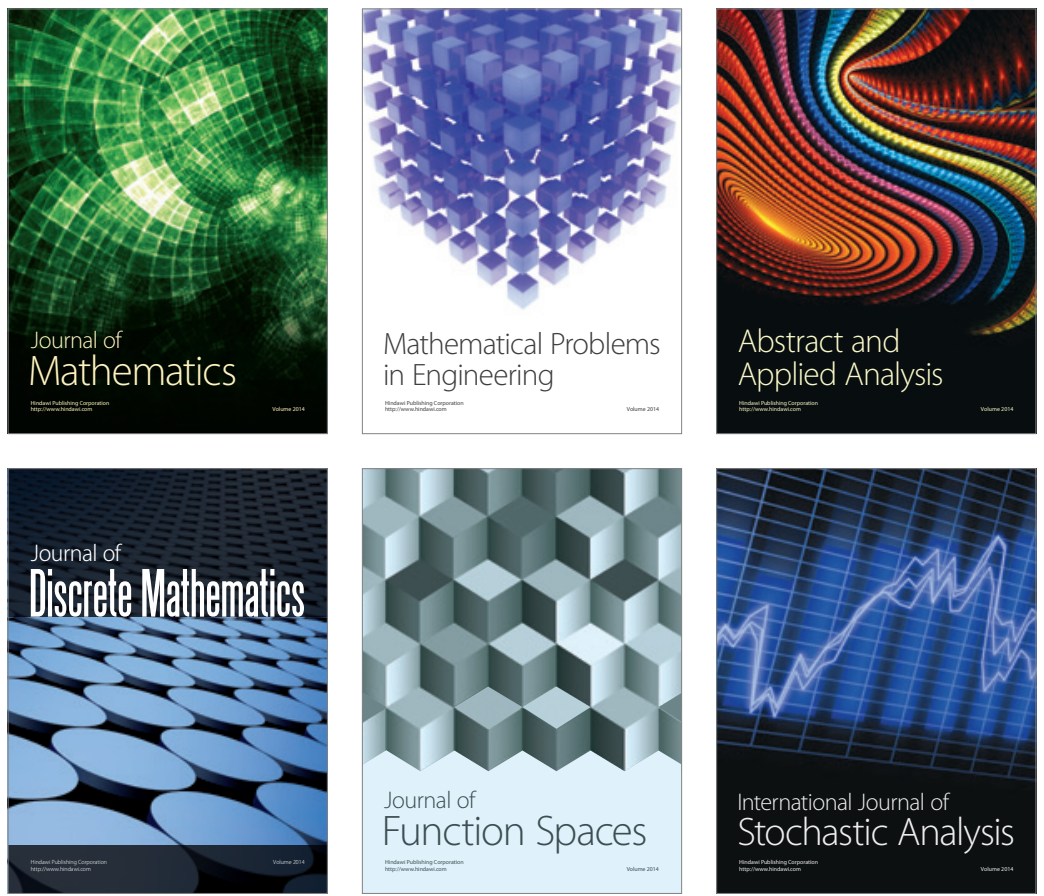

Journal of

Function Spaces

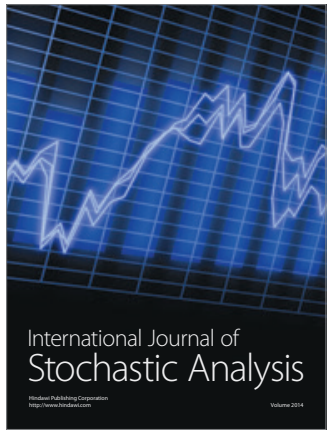

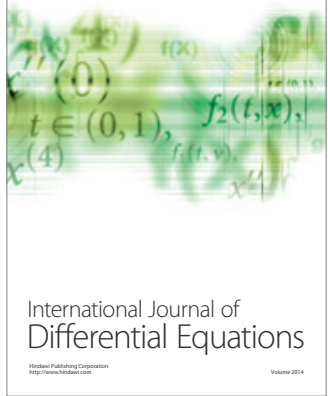
